\title{
Exciton condensation in quantum wells
}

\author{
V.I. Sugakov \\ Institute for Nuclear Research, 47 Nauki Ave., Kiev, 03680, Ukraine \\ E-mail: sugakov@i.com.ua
}

Received May 24, 2006, revised July 7, 2006

\begin{abstract}
The theory of exciton condensation is given in two-dimensional systems under suggestion that condensation occurs in really space and condensed phase arises as a result of an attractive interaction between excitons. Due to the finite value of exciton lifetime the sizes of exciton condensed phase regions are restricted and the condensed phase appears in a form of system of islands amid exciton gas. The joint solution of kinetic equations for island size and exciton diffusion equation in the space between islands has been obtained. The theory is applied to explanation of experimental manifestation of condensed phase in quantum wells and also to explanation of the periodical fragmentation, which was observed in luminescence spectrum from a ring around a laser spot in a crystal with double quantum wells. For such explanations the theory does not require the exciton Bose-Einstein condensation.
\end{abstract}

PACS: 73.21.Fg, 78.67.De

Keywords: two-dimensional systems, exciton condensation theory, luminescence spectrum periodical fragmentation.

\section{Introduction}

There were many attempts to observe exciton condensed phase. Stirring problem is the problem of Bose-Einstain condensation of excitons [1,2] with its interesting physical consequences: superfluidity, narrowing of optical bands and others. But usually electron-electron correlation energies in condensed phase are larger than energy of interaction between excitons and consequently excitons lose their individuality and well known and well good studied state of electron-hole liquid is created [3]. The creation of electron-hole liquid phase was observed and studied in germanium, silicon and other materials [4-7].

The crystal $\mathrm{Cu}_{2} \mathrm{O}$ was considered for some time as perspective system for exciton Bose-Einstein condensation because the exciton in $\mathrm{Cu}_{2} \mathrm{O}$ has small radius and large value of binding energy. But due to Auger decay processes it is difficult to create in this crystal the exciton density that satisfies Bose-Einstein condencation criterion [8]. So the observation of Bose-Einstain condensation is not conformed in bulk materials.

Recently intense investigations of excitons under intensive irradiation were carried out for indirect excitons in quantum wells (QWs) [9-16]. Indirect excitons in double QW structures with electrons and holes driven to the separate wells by an electric field are long living particles. The enhanced lifetime makes it possible to create large concentrations of excitons and study effects of the exciton-exciton interaction and a possibility of an exciton condensation, in particular. The authors [9] observed an appearance of narrow line in luminescence spectra in the crystal with double quantum well at some threshold value of pumping. In AlGaAs and InGaAs based structures, a nontrivial feature in the photoluminescence spectra of indirect excitons was observed [11-16]. A spot of the laser excitation was reported to be surrounded by a concentric bright ring separated from the laser spot by an annular dark intermediate region. The distance between the ring and the spot grew with increasing the intensity of the pumping and reached up to hundreds $\mu \mathrm{m}$ exceeding by much the exciton diffusion path. At low temperatures, the external ring fragmented into a structure with a strongly evaluated periodicity on a macroscopic scale $[11,12,15]$.

\section{Model of system. Basic equations}

In the paper a model of exciton condensation is developed which is based on the traditional phase transition theory, generalized for the case of a nonequilib- 
rium system, in which particles (excitons) have finite lifetime. The condensed exciton phase is assumed to exist, and its density is higher than that of the gaseous phase. In this case, the condensation takes place in real space, not in momentum space at $\mathbf{k}=0$, i.e., the condensed phase is not the Bose-Einstein condensate. The reasons for the application of the traditional phase transition theory for the description of the exciton system are following:

Real QWs comprise defects of different types. Even at perfect boundaries in semiconductor alloys like $\mathrm{Ga}_{1-x} \mathrm{Al}_{x}$ As there are always composition fluctuations either in the well or in the barrier, or both. The authors of [17] showed that the disorder sufficiently toughens the criteria for the appearance of quantum coherence effects. Disorder leads to broadening of luminescence line. For the line, which the authors $[9,10,15]$ connect with the condensed phase, the width is $Q=0.1-1 \mathrm{meV}$. According to the calculation [17], for $Q=0.5 \mathrm{meV}$ the condensed phase can appear only for the densities more than $2.5 \cdot 10^{11} \mathrm{~cm}^{-2}$. In the case of the smallest disorder observed in experiment $(Q=0.1 \mathrm{meV})$ at the exciton density $5 \cdot 10^{10} \mathrm{~cm}^{-2}$, the Kosterlitz-Thouless transition happens at $T<0.4 \mathrm{~K}$, which is significantly lower than the critical temperature in the absence of disorder. The understanding of the toughening of the quantum criteria is possible from the following argumentation. The presence of disorder gives rise to the exciton scattering with wave vector changing in the well plane, and this causes broadening of the exciton emission line width $Q$. The free path time $\tau$ can be estimated from the relation $\tau Q \approx \hbar$. Hence, for the line width $Q=0.1-1 \mathrm{meV}$ the time of the free path equals to $0.7-7 \mathrm{ps}$, and for the average exciton velocity corresponding to $T=1 \mathrm{~K}$ the free pass length $v \tau \approx 7-70 \mathrm{~nm}$. Therefore, for the exciton density of $10^{10}-10^{11} \mathrm{~cm}^{-2}$, the wave function loses its coherence due to the scattering on defects at the distances smaller than the distance between excitons. This makes doubtful the explanation by means of cooperative quantum effects the phenomena observed at $T>1 \mathrm{~K}$ $[11,15]$. So, on the one hand, the condition for exciton collective quantum effects is facilitated in comparison with the condition for the system of alkaline metal atoms, in which Bose condensation was observed [18], because the exciton mass is sufficiently smaller than atom mass. On the other hand, these conditions become tougher, because of the presence of random forces due to such external to the system factors as the spatial disorder, the interaction with phonons, etc.

For the formation of the condensed state in real space, the attractive interaction between excitons is required. At the same time, owing to the presence of a dipole moment, directed perpendicularly to the well plane, repulsive interaction is realized for indirect excitons at large distances between them. However, at close distances, the exchange interaction between electrons and between holes has the essential contribution. It is known that in a single $\mathrm{QW}$ bases on AlGaAs, a biexciton exists with a relatively large binding energy (23\%) of the exciton binding energy [19]. The exchange interaction between the electrons and between holes of two excitons, located in the same well, takes place in the double QW, too. Therefore, one has to expect an attraction between excitons located at close distances (several exciton radii) at not very large distances between QWs, when the dipole moments of excitons are not very large. Thus, the calculations [20,21] of multiexciton system of indirect excitons taking into consideration exchange energy, Van-der-Waals energy and correlation energy showed that the energy of the system as the function of the density has a minimum at nonzero exciton density for not very separated quantum wells $(d<1.1 a$, where $d$ is the distance between the wells, $a$ is the exciton radius in the well), when the dipole-dipole repulsion is not too large (see Fig. 2 in the [21]). In this case, the appearance of excitonic liquid state become possible in a double QW. The authors also showed that in these conditions, the excitonic liquid is more advantageous energy-wise than the formation of biexcitons. Using the parameters of GaAs crystal and the results of paper [21], it is can be shown that the binding energy per exciton is negative (i.e., there is an attractive interaction) at the exciton density of order of $(1.1-2.5) \cdot 10^{11} \mathrm{~cm}^{-2}$. This value is the exciton density in the condensed phase. In the model considered here, the system consists of both condensed and gas phase regions. At critical parameters of system the fraction of condensed phase islands is small. So, the critical density is determined by the mean value of the density, which is of several times smaller than the condensed phase density. Therefore, the theory predicts the realistic value of the density of several units of $10^{10} \mathrm{~cm}^{-2}$, at which the condensation can occur.

Besides the phase of a dielectric liquid phases with different polarization can be realized in double QWs. The phase diagrams of these phases are presented in [22].

The sizes of condensed phase regions are restricted both from above and from below. The radius of new phase nucleus must be larger some threshold which is determined by a surface energy. A presence of the surface energy determines the shape of condensed phase, in two-dimensional case the condensed phase has disk-like shape. A restriction of the size from above caused by nonequilibrium system which is connected with exciton creation and exciton decay. In some range of pumping a number of excitons created by 
light in unit time inside of condensed phase region is less than the number of excitons which disappear. In this case the steady state of the system is possible if losses of excitons inside of condensed phase compensates by an exciton inflow from surrounding region of exciton gas phase. Such compensation is not possible at large radius of exciton condensed phase. As the result in the two-dimensional case the condensed phase must exist as a system of islands similar to electron-hole drops in bulk semiconductors. The condition of free energy minimum is satisfied if the islands have the form of disks. In studied system the islands should be placed on the ring where the exciton density has maximum. Since islands of condensed phases takes excitons from the same source, two islands can not be situated close one to another. Moreover, the distance between islands can not be large because in this case the exciton density between them becomes greater than a critical value and as the result a new island may appear. So, there is specific interaction between condensed phases through the exciton concentration fields and as a result there is optimal distance between islands.

To study the creation and structure of the exciton condensed phase we used the kinetic equations which describe a nucleation and growth of a new phase. Such theory was suggested for a description of a liquid phase formation from supersaturated gas, spinodal decomposition dynamics [23] and electron-hole drops in semiconductors [24-27]. There are several original peculiarities of the presented here paper, namely: 1) the consideration of two-dimensional problem, 2) taking into account the mutual influence of condensed phase islands, 3) the investigation of nonuniform particle distribution, 4) taking into account the finite value lifetime and the pumping of excitons.

The distribution of exciton density at a steady state irradiation will be determined from a joint solution of the system of equations for the size distribution function of islands and the exciton diffusion outside islands. The size of the islands are determined by four processes: the creation of excitons by the pumping, the capture of the excitons from environment, an escape of excitons from the island, and the exciton decay. Let us introduce the distribution function $f_{n}$, which determines the probability of the island to have $n$ excitons. The distribution function satisfies the following kinetic equation:

$$
\frac{\partial f_{n}}{\partial t}=-j_{n+1}+j_{n},
$$

where $j_{n}$ is the probability current for transitions between island states with $n$ and $(n-1)$ excitons, while $j_{n+1}$ stands for transitions between the states with $(n+1)$ and $n$ excitons,

$$
j_{n}=W^{(-)}(n) f_{n}-W^{(+)}(n-1) f_{n-1},
$$

$W^{( \pm)}$is the probability of transition in unit time with increasing (decreasing) number of excitons on unity. The values will be determined later.

We shall study the steady-state solutions of Eq. (1). There are two types of stationary solutions. In the first case $j_{n}=j_{n+1}=j=$ const. In the theory of phase transitions this solution is used for a determination of a rate of phase nucleation. In the second case

$$
j_{n}=j_{n+1}=0 .
$$

The solution under the condition (3) describes the dynamic equilibrium state which is formed after some time of pumping action. Such type solution we will investigate in future. Setting $\partial f_{n} / \partial t=0$ and $j_{n}=0$ in (2) we obtain the following steady-state solution:

$$
f_{n}=f_{1} \exp \left(\sum_{m=1}^{m=n} \ln \frac{W^{(+)}(m-1)}{W^{(-)}(m)}\right) .
$$

As we shall see the distribution function has sharp maximum at some value of $n$, which is much larger than unity $(n \gg>1)$. Islands with a large number of excitons and with a shape of disks are described by radiuses $R_{n}=(n / \pi)^{1 / 2}$ instead of $n$. In this case the probabilities of $W^{(+)}(n)$ и $W^{(-)}(n)$ are satisfy the following conditions:

$$
\begin{gathered}
W^{(+)}(R)=2 \pi R c(R) W_{f i}(R)+\pi R^{2} G_{n}, \\
W^{(-)}(R)=2 \pi R c_{i} W_{i f}(R)+\pi R^{2} c_{i} / \tau_{\mathrm{ex}},
\end{gathered}
$$

where $W^{( \pm)}(R) \equiv W^{( \pm)}(n), W_{f i}$ and $W_{i f}$ are the probabilities for the exciton to be captured by the disk and to escape from the disk per unit length of the circle in unit time and per one exciton of disc circle, respectively, $G_{n}=\left\lceil G(r) \mathrm{d} \mathbf{S}_{n} / \pi R_{n}^{2}\right.$ is the mean value of the exciton pumping $G(r)$ over the $n$th island area, $c(R)$ and $c_{i}$ are the exciton densities on the circle of the disk and inside the disk.

The relationship between the transition probabilities $W_{f i}$ and $W_{i f}$ may be obtain using the detailed balance principle. In the case of infinite exciton lifetime and absence of pumping the equilibrium state between condensed phases is formed due to processes of exciton exchange between the island and an environment. Then in an equilibrium state the following condition should take place

$$
W_{f i}(R) c_{\mathrm{eq}}=W_{i f} c_{i} .
$$


Where $c_{\mathrm{eq}}$ is the density of exciton gas which is in equilibrium state with the island. The Eq. (7) can be rewritten in the form

$$
\frac{W_{i f}(R)}{W_{f i}(R)}=\frac{W_{i f}(\infty)}{W_{f i}(\infty)} \exp \left(\frac{\alpha_{0}}{c_{i} R k T}\right),
$$

where $W_{f i}(\infty)$ and $W_{i f}(\infty)$ are the transition probabilities in the case of a straight line boundary between condensed and gas phases, $W_{f i}(\infty) / W_{i f}(\infty)=c_{\infty} / c_{i}$, $c_{\infty}$ is the equilibrium concentration of excitons on the straight division line

$$
c_{\infty}=c_{10} \exp (-\varphi / k T),
$$

$\varphi$ is the energy condensation per one exciton, $c_{10}=$ $=\gamma\left(m^{*} k T / 2 \pi \hbar^{2}\right), m^{*}$ is the effective exciton mass, $\gamma$ is the degeneracy of the exciton state, $\alpha_{0}$ is the surface strain.

The calculation of these probabilities needs an application of quantum-mechanical calculations. For an estimation of these values we shall calculate the exciton flux from gas phase in direction of the condensed phase. To penetrate in an island exciton should overcome barrier caused by a dipole-dipole repulsion between excitons.

Hereinafter we introduce the radius distribution function $f(R)$ instead of the particle distribution function $f_{n}$ by the condition $f(R)=f_{n} d n / d R=2 \pi R c_{i} f_{n}$. Let us introduce the dimensionless variables: $\widetilde{R}=$ $=R\left(c_{i}\right)^{1 / 2}, \tilde{c}(\tilde{R})=c(R) / c_{i}, v_{f i}=W_{f i} \tau_{\mathrm{ex}}\left(c_{i}\right)^{1 / 2}, \tilde{G}=$ $=G \tau_{\text {ex }} / c_{i}$. After some transformations the radius distribution function (4) may be presented in the form

$$
f(\tilde{R})=f_{b} \exp (s(\tilde{R})),
$$

where

$$
s(\tilde{R})=2 \pi \int_{\tilde{R}_{S}}^{\tilde{R}} \ln \left(\frac{2 \tilde{c}(\tilde{R}) v_{f i}+\tilde{R} \tilde{G}}{2 v_{f i} c_{10} \exp (-(\varphi-\alpha / \tilde{R}) / T)+\tilde{R}}\right) \tilde{R} d \tilde{R},
$$

$$
f_{b}=f_{1} \exp \left(\sum_{m=1}^{n_{S}} \ln \frac{W^{(+)}(m-1)}{W^{(-)}(m)}\right),
$$

where $n_{s}$ is the value of order of several unit $\left(n_{s} \leq 10\right), n_{s}=\pi R_{s}^{2} c_{i}$. The factor $f_{b}$ marked out specially since the sum in exponent of it can not be replaced by integral.

In the stationary case, the equation for the exciton density $c(\tilde{R})$ in the region outside the islands satisfies the diffusion equation

$$
D_{\mathrm{ex}} \Delta_{2} c(r)-\frac{c(r)}{\tau_{\mathrm{ex}}}=-G,
$$

where $D_{\text {ex }}$ is the exciton diffusion coefficient.

The solution of the equation (13) should satisfy the following boundary conditions: the current of excitons to every island should be equal to the difference between the number of excitons captured by the island and the number of excitons which escape from the island. So, the boundary conditions on $i$ th disk has the form

$$
\left.2 \pi R_{i} D_{\mathrm{ex}}(\nabla c(\mathbf{r}) \cdot \mathbf{n})\right|_{i}=\left(\left.2 \pi R_{i}\left(W_{f i} c(\mathbf{r})-W_{i f} c_{i}\right)\right|_{i}\right),
$$

where $R_{i}$ is the radius of the $i$ th island, $\mathbf{n}$ is the outward and unitary normal vector to the circular boundary of the $i$ th island, the notation $I_{i}$ means that the boundary conditions of Eq. (14) are applied at the surface of an island.

The solution of equation (12) may be written in the following form:

$c(\mathbf{r})=n_{\mathrm{ex}}(r)+\sum_{i} \sum_{m=0}^{\infty} A_{i m} K_{m}\left(\left|\mathbf{r}-\mathbf{r}_{i}\right| \gamma l\right) \cos \left(\varphi_{i}(r) m\right)$,

where

$$
n_{\mathrm{ex}}(r)=\frac{1}{2 \pi D_{\mathrm{ex}}} \int K_{0}\left(\left|\mathbf{r}-\mathbf{r}^{\prime}\right| l\right) G\left(\mathbf{r}^{\prime}\right) d S^{\prime},
$$

$K_{m}(x)$ is the modified Bessel function, $\mathbf{r}_{i}$ is the position of the center of the $i$ th island, $\varphi_{i}(r)$ is the polar angle of the point $\mathbf{r}$ in the coordinate system with the origin at the center of the $i$ th island, $l=\left(D_{\mathrm{ex}} \tau_{\mathrm{ex}}\right)^{1 / 2}$ is the exciton diffusion length. The first term in the expression (15) is the particular solution of the inhomogeneous equation (13). The second term is the solution of the homogeneous equation (13). So, the expression (15) satisfies the equation (13). The unknown coefficients $A_{\text {im }}$ are to be determined from the boundary conditions (14). The solution (15) has distinct meaning: the first term describes the exciton density created by pumping in the case of absence of condensation phases, the second term describes the perturbation of the exciton density caused by presence of condensed phase islands which capture excitons.

\section{Uniform pumping}

In this section we shall investigate an uniform creation of excitons in double quantum well [28], i.e., the pumping does not depend on space coordinates. In this case the first term in (15) equals

$$
n_{\mathrm{ex}}=G \tau_{\mathrm{ex}} .
$$

Firstly we consider the behavior of the system in a vicinity of threshold of condensed phase formation. In this case the mutual influence between islands can be neglected and single island may be studied. The solu- 
tion (15) gives together with boundary condition (14) the following dependence of exciton density outside of island with a center in the origin of coordinates (in dimension units)

$$
\tilde{c}(\tilde{\mathbf{r}})=\tilde{G}+A_{00}^{S}(\tilde{R}) K_{0}(|\tilde{\mathbf{r}}| / \tilde{l}),
$$

where

$$
A_{00}^{S}(\tilde{R})=-\frac{\nu\left[\tilde{G}-\tilde{c}_{10} \exp (-(\varphi-\alpha / \tilde{R}) / T)\right]}{K_{1}(\tilde{R} / \tilde{l}) \tilde{l}+v K_{0}(\tilde{R} / \tilde{l})} .
$$

The radius distribution function obtained from (10) for different pumping is presented in Fig. 1. The function has sharp maximum for exception of the pumping very closed to the threshold value. The maximum determines the most probable meaning of radius of the island. The threshold value of pumping can be obtained from two conditions:

$$
\partial f(\tilde{R}) / \partial \tilde{R}=\partial^{2} f(\tilde{R}) / \partial \tilde{R}^{2}=0 .
$$

We calculated the phase diagram «critical pumping -temperature» using the formulae (10), (20). Parameters $\left(m^{*}, D_{\mathrm{ex}}, \tau_{\mathrm{ex}}\right)$ were chosen for GaAs, $\varphi, \alpha, T_{d}$ were fitting parameters. Results are presented in Fig. 2, where points are experimental data from [9]. It is seen that theory describes the experiments without suggestion about Bose-Einstein condensation.

Many islands arise with increasing pumping. There is specific interaction between islands through exciton concentration fields. In studied system the islands should be localized on the ring where the exciton density has maximum. Since islands of condensed phases takes excitons from the same source, two islands can not be situated close one to another. Moreover, the distance between islands can not be large because in this case the exciton density between them becomes greater than a critical value and as the result a new is-

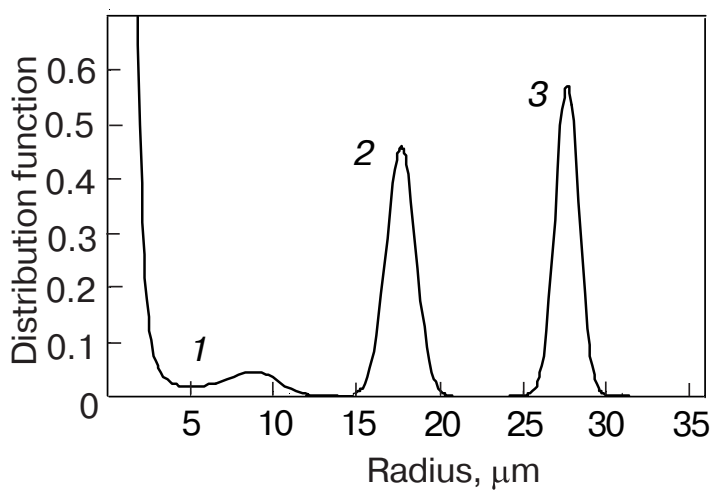

Fig. 1. Radius distribution function for single condensed phase island in quantum well at different pumping $\tilde{G}$ : 0.00715 (1), 0.01 (2), 0.015 (3). $\varphi=10 \mathrm{~K}, a=4 \mathrm{~K}, T=2 \mathrm{~K}$, $\tilde{l}=320, c_{i}=10^{11} \mathrm{~cm}^{-2}$.

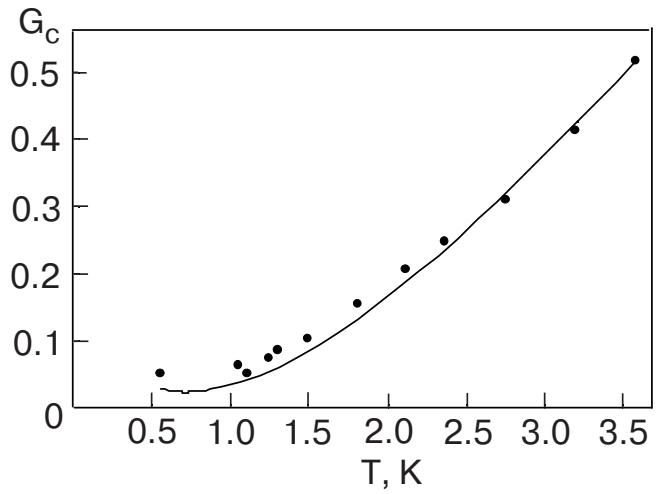

Fig. 2. The dependence of threshold value of pumping on temperature (solid curve). $\varphi=3 \mathrm{~K}, \alpha=1 \mathrm{~K}, T_{d}=2.5 \mathrm{~K}$, $\tilde{l}=100, \tau_{\mathrm{ex}}=10^{-8} \mathrm{~s}$. The points correspond to the experimental data from [9].

land may appear. Thus, there is optimal distance between islands.

The connection between islands occurs due to the dependence of the exciton density $c(\tilde{R})$ in (11) at the surface of the considered island versus the presence of other islands. We shall make two approximations in further calculations. We shall suggest that the distance between islands is larger than the island radius. Under such condition the exciton density perturbation caused by the presence of some island slowly changes in limits of a size of other island and this perturbation may be considered as constant in the limits of island size. It means that only terms with $m=0$ gives the contribution in the sum of the expression (15). So, $A_{\text {im }}=0$ at $m \neq 0$. Also we suggest that this perturbation is formed by many islands and as result it may be studied in a meanfield approximation. Let us consider the exciton density in vicinity of some island, for example, with $i=0$. In mean field approximation all coefficients $A_{i 0}$ in the sum of (15) can be replaced by their average value $\bar{A}_{i 0}$ in all terms for exception of the term with $i=0$, the sum over $i$ can be changed by integral in a suggestion that islands are distributed in space with density $c_{\text {isl }}=N / S$, where $N$ is the number of islands, $S$ is the area of quantum well. Such approximation allows to simplify the boundary condition (14). After some transformations from boundary conditions (14) we obtain the following expressions for exciton density on the surface of the island with the radius $\tilde{R}$ :

$$
\begin{gathered}
\tilde{c}(\tilde{R}) \rightarrow \tilde{c}\left(\tilde{R}, \tilde{c}_{\mathrm{isl}}\right)=\tilde{G}+A_{00}\left(\tilde{R}, \overline{\tilde{R}}, \tilde{c}_{\mathrm{isl}}\right) K_{0}(\tilde{R} / \tilde{l})+ \\
+2 \pi \bar{A}_{00}\left(\overline{\tilde{R}}, \tilde{c}_{\mathrm{isl}}\right) \tilde{l}^{2} \tilde{c}_{\mathrm{isl}},
\end{gathered}
$$

where 


$$
A_{00}\left(\tilde{R}, \overline{\tilde{R}}, \tilde{c}_{\mathrm{isl}}\right)=-\frac{v\left(\tilde{G}+2 \pi \bar{A}_{00}\left(\overline{\tilde{R}}, \tilde{c}_{\mathrm{isl}}\right) \tilde{l}^{2} \tilde{c}_{\mathrm{isl}}-\tilde{c}_{10} \exp \left(-\left(\varphi_{0}-\alpha_{0} / \tilde{R}\right) / T\right)\right)}{K_{1}(\tilde{R} / \tilde{l}) \tilde{l}+v K_{0}(\tilde{R} / \tilde{l})},
$$

$\bar{A}_{00}\left(\overline{\tilde{R}}, \tilde{c}_{\mathrm{isl}}\right)=-\frac{v\left(\tilde{G}-\tilde{c}_{10} \exp (-(\varphi-\alpha / \overline{\tilde{R}}) / T)\right)}{\tilde{l} K_{1}(\overline{\tilde{R}} / \tilde{l})+v K_{0}(\overline{\tilde{R}} / \tilde{l})+2 \pi v \tilde{l}^{2} \tilde{c}_{\mathrm{isl}}}$,

$\overline{\tilde{R}}$ is the mean radius, which coincides with most probable one.

Let us consider the ensemble of identical systems in which may be realized the state with different number of islands. In system with many islands the distribution function depends on radiuses (or numbers of excitons) of all islands and determined by the function $f\left(\tilde{R}_{1}, \tilde{R}_{2}, \ldots, \tilde{R}_{i}, \ldots, \tilde{R}_{N} ; N\right)$. The equation (1) should be rewritten for many-islands system. In the model of self-consistent field the distribution function can be present as product of distribution functions for separate islands

$$
f\left(\tilde{R}_{1}, \tilde{R}_{2}, \ldots, \tilde{R}_{i}, \ldots, \tilde{R}_{N} ; N\right) \approx \exp \sum_{i=1}^{N} s_{\text {isl }}\left(\tilde{R}_{i}, \tilde{c}_{\text {isl }}\right) .
$$

After integrating of this value over radiuses of all islands we obtain the probabilities of a realization of the state with $N$ islands (or the probability of the state with the density $\tilde{c}_{\text {isl }}$ )

$$
F\left(\tilde{c}_{\mathrm{isl}}\right) \approx \exp \left(S \tilde{c}_{\mathrm{isl}} S_{\mathrm{isl}}\left(\overline{\tilde{R}}, \tilde{c}_{\mathrm{isl}}\right)\right)
$$

$$
s_{\text {isl }}\left(\overline{\tilde{R}}, \tilde{c}_{\text {isl }}\right)=2 \pi \int_{R_{s}}^{\overline{\tilde{R}}} \ln \left(\frac{2 \tilde{c}(\tilde{R}) v_{f i}+\tilde{R} G}{2 v_{f i} c_{10} \exp (-(\varphi-\alpha / \tilde{R}) / T)+\tilde{R}}\right) \tilde{R} d \tilde{R} .
$$

The most probable density of islands is determined by the condition

$$
\frac{\partial\left(c_{\mathrm{isl}} S_{\mathrm{isl}}\left(\overline{\tilde{R}}, \tilde{c}_{\mathrm{isl}}\right)\right)}{\partial \widetilde{c}_{\mathrm{isl}}}=0 .
$$

Intensity of the emission, created by excitons of condensed phase is proportional to the number of excitons, which islands contain

$$
I_{T} \propto \pi \overline{\tilde{R}}^{2} \tilde{c}_{\text {isl }} .
$$

In approximation (28) we neglect by the stimulating emission.

The results of temperature dependence calculation of emission intensity according (28) are presented in the Fig. 3. The dependence is described by straight line. The line crosses the axis at the temperature for which the considered pumping has threshold value. Such behavior of intensity coincides with empirical formula obtained from experiment in the papers [9]

$$
I_{T} \propto 1-\frac{T}{T_{c}} .
$$

So, we have explained the experiments without suggestion about Bose-Einstein condensation of excitons.

\section{Formation of luminescence from the ring outside the laser spot}

As it was mentioned in Introduction, the authors of [11-16] observed the emission from the ring outside the laser spot at the distances from a center of the spot much larger than exciton diffusion length. At low temperatures, the ring fragmented into a periodical structure $[11,12,15]$.

The mechanism of ring appearance was suggested [15,16] basing on two assumptions: 1) without light irradiation the well is populated with a certain density of electrons; 2) holes are captured by the well with a

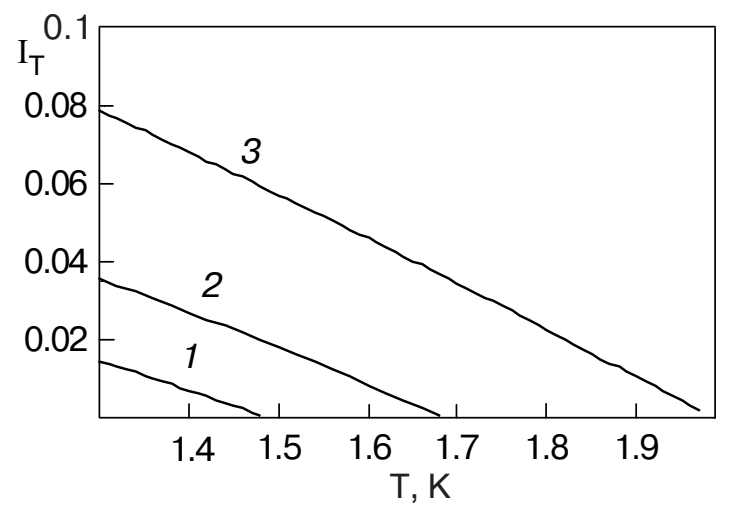

Fig. 3. The dependence of luminescence intensity on temperature at different pumping, $\tilde{G}: 0.085$ (1), 0.11367 (2), 0.1644 (3). Parameters are the same as in Fig. 2. 
larger probability than electrons. As the result, the irradiated structure develops two differently charged spatial regions. The laser spot and the region around it are charged positively while the region far away remains negative. Electrons and holes can recombine only where they meet, therefore, the sharp luminescence ring is formed on the boundary between regions of opposite charges.

The model of the fragmentation observed in $[11,12,15]$ was bild in $[29,30]$ on the base of the traditional phase transition theory, generalized for the case of a nonequilibrium system, in which particles (excitons) have finite lifetime. The recombination of electrons and holes on the ring determines the exciton pumping. According to $[15,16]$ the exciton density has sharp maximum at the some distance from laser spot. If the density exceeds the threshold value the islands of condensed phase are generated on the ring (see insert in Fig. 4). The system can be described by kinetic equations (1) with interaction between islands through exciton concentration fields. The study of sizes of islands (fragments) and the distance between them is similar to the consideration of island parameters at uniform pumping presented above. Results of such study are presented in $[29,30]$. The probability for system to have $N$ islands is described by the formula

$$
f(N)=f_{0 \text { isl }} \exp \left(S_{\text {isl }}(N)\right) .
$$

The most probable number of islands is determined by extremum of function $S_{\text {isl }}(N)$. The results of taken from $[29,30]$ calculations of temperature dependence of island radius, distance between islands and fluctuations of the distances are presented in Fig. 4. The distance from the laser spot to fragmented ring $\left(r_{0}\right)$ equals $200 \mu \mathrm{m}$.

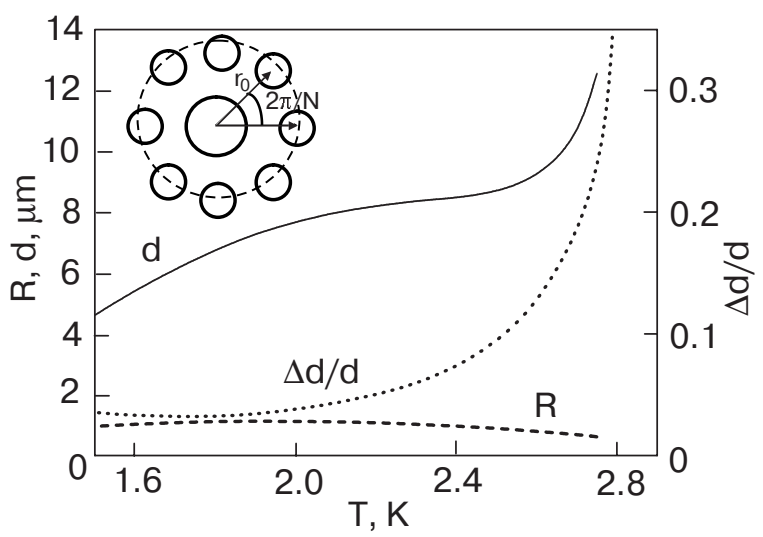

Fig. 4. Dependences of the island radius $R$, distance between islands $d$ and ratio of the island fluctuation shift to the distance between islands $\Delta d / d$ on temperature. The insert shows positions of exciton condensed phase islands around the laser excitation spot.
In this section we shall consider the case when the condensed phase of excitons is shaped as a ring around the laser spot in region of the largest electron-hole recombination. Let the internal and external radii of the ring formed by the condensed phase are equal to $r_{1}$ and $r_{2}$, respectively, and $r_{0}$ is the radius of the maximum of the exciton production rate $G(r)$ (see the insert in Fig. 5). The ring grows due to the exciton creation by pumping and due to the exciton inflow from the environment. The ring narrows as a result of the exciton decay and the exciton escape.

The consideration of the exciton condensation in the shape of a ring is similar to the study of the formation of exciton islands considered in the previous sections. Therefore, we shall set out the problem briefly. Similarly to the consideration of the islands, let us introduce the distribution function $f_{n}$, which determines the probability that the condensed phase shaped as a ring contains $n$ excitons. The distribution function obeys to an equation similar to the equation (1), in which the probability current is equal to

$$
\begin{gathered}
j_{n}=2 \pi W_{f i}\left(r_{1, n-1} c\left(r_{1, n-1}\right)+r_{2, n-1} c\left(r_{2, n-1}\right)\right) f_{n-1}+ \\
+2 \pi \Delta r_{n-1} r_{0} \bar{G}\left(r_{n-1}\right) f_{n-1}-2 \pi W_{i f}\left(r_{1 n}+r_{2 n}\right) c_{i} f_{n}- \\
-\pi\left(r_{2, n}^{2}-r_{1, n}^{2}\right) c_{i} f_{n} / \tau_{\mathrm{ex}},
\end{gathered}
$$

ćwhere $c\left(r_{1, n}\right)$ and $c\left(r_{2, n}\right)$ are the exciton density on the internal and the external circular boundaries of the ring, $\Delta r_{n}=r_{2, n}-r_{1, n}$ is the thickness of the ring, $\bar{G}\left(r_{n}\right)$ is the mean value of the pumping

$$
\bar{G}\left(r_{n}\right)=\int_{r_{1, n}}^{r_{2} n} r G(r) d r /\left(\Delta r_{n} r_{0}\right) .
$$

Other designations have been defined in the previous sections.

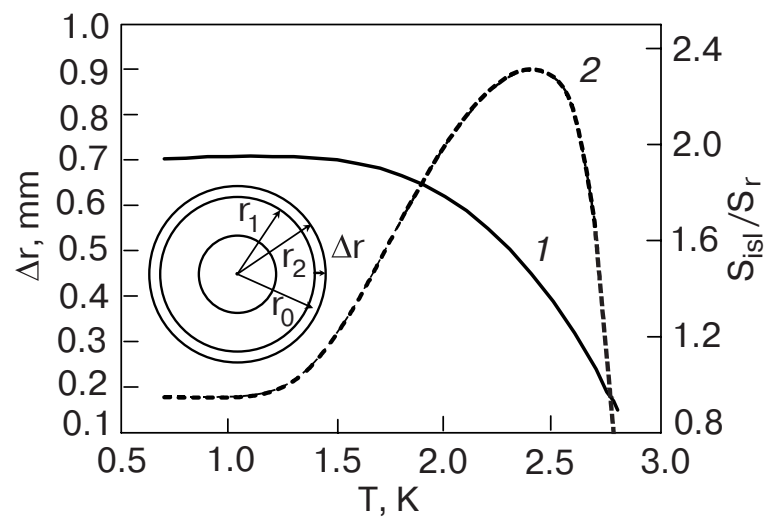

Fig. 5. Dependences of the thickness of condensed phase ring $\Delta r$ (1) and ratio of values $S_{\mathrm{isl}}$ and $S_{r}$ (2) on temperature. The insert shows the condensed phase in the form of a ring. 
Further we take into account the fact that $n \gg 1$ and obtain a Fokker-Planck equation for the distribution function. We assume also that the thickness of the ring is narrower than the ring radius $r_{0} \gg \Delta r_{n}$. At such approximation $c\left(r_{1, n}\right) \approx c\left(r_{2, n}\right)$.
After reductions similar to those carried out in the subsection 2, we obtain the following expression for the distribution function:

$$
f_{n}=f_{0} \exp \left(\int_{0}^{n} 2 \frac{2\left(W_{f i} c\left(r_{1, n}\right)-W_{i f} c_{i}\right)-\Delta r\left(c_{i} / \tau_{\mathrm{ex}}-G\left(r_{n}\right)\right.}{2\left(W_{f i} c\left(r_{1, n}\right)+W_{i f} c_{i}\right)-\Delta r\left(c_{i} / \tau_{\mathrm{ex}}+G\left(r_{n}\right)\right.}\right) d n
$$

Coefficients $W_{f i}$ and $W_{i f}$ are connected by the detailed balance principle of Eq. (8). As the radius of the ring is significantly larger than the radius of an island of the previous section we may neglect the surface energy and put $\alpha_{0}=0$.

The exciton density beyond the region of the condensed phase obeys Eq. (13). Under condition of the accepted approximation that the thickness of the ring is significantly smaller than its radius, the solution of this equation takes the form

$$
c(r)=n_{\mathrm{ex}}+c_{s 0} \exp (-r / l),
$$

where $n_{\mathrm{ex}}$ is the solution of the nonuniform Eq. (13). It describes the exciton density created by the electron-hole recombination in the absence of the condensed phase. The value of $c_{s 0}$ is determined by the boundary condition of Eq. (14). For the ring, this condition may be written in the form

$$
D_{\mathrm{ex}} \frac{\partial c(r)}{\partial r}=W_{f i} c\left(r_{1, n}\right)-W_{i f} c_{i} .
$$

The solution of Eq. (33) with the boundary condition of Eq. (34), assuming that the thickness of the ring of the condensed phase is smaller than the diffusion length $(\Delta r<l)$, reads

$$
c\left(r_{1, n}\right)=\frac{n_{\mathrm{ex}}-c_{10} \exp (-\varphi / T)}{1+W_{f i} l / D_{\mathrm{ex}}}+c_{10} \exp (-\varphi / T) .
$$

Further we shall use the thickness distribution function which is defined as $f(\Delta r)=f_{n} d n / d \Delta r=2 \pi r_{0} c_{i} f_{n}$. Using dimensionless variables, we obtain the following formula for the thickness distribution function from Eqs. (32) and (35):

$$
f(\Delta r)=f_{0 r} \exp \left(S_{r}(\Delta r)\right)
$$

where

$$
S_{r}(\Delta r)=\int_{0}^{\Delta r} \frac{4 \pi r_{0}\left\{2 v\left[\bar{n}_{\mathrm{ex}}-c_{10} \exp (-\varphi / T)\right]-\Delta r^{\prime}\left(1-G\left(r_{0}\right)(1+v / l)\right\} d \Delta r^{\prime}\right.}{2 v\left[\bar{n}_{\mathrm{ex}}-c_{10} \exp (-\varphi / T)\right]+\Delta r^{\prime}\left(1+G\left(r_{0}\right)\right)(1+v / l)+4 v c_{10}(1+v / l) \exp (-\varphi / T)} .
$$

The most probable thickness of the ring is determined by the condition

$$
\frac{\partial S_{r}(\Delta r)}{\partial \Delta r}=0 .
$$

Using Eq. (38), we have calculated the most probable thickness of the ring if the condensed phase is realized in the shape of a ring at the following values of parameters: $\varphi=10 \mathrm{~K}, \alpha_{0}=3 \mathrm{~K}, c_{i}=10^{11} \mathrm{~cm}^{-2}$, $m^{*}=0.37 m_{0}, \tau_{\mathrm{ex}}=10^{-7} \mathrm{~s}$. The same values of parameters were used in [30] during analysis of a fragment formation. As seen from Fig. 5, the thickness of the ring decreases with rising the temperature.

The question to answer is what type of the condensed phase is realized? Is it the fragmented ring or is it the continuous ring? In order to find the solution of the problem we have performed the following estimations. We have compared the probabilities of the appearance of these two states. The curve 2 in Fig. 5 shows the ratio of the values $S_{\text {isl }}$ and $S_{r}$, which determine the probabilities according to the formulas of Eqs. (30) and (36), correspondingly. It should be noted that absolute values of $S_{\text {isl }}$ and $S_{r}$ are large for exception the region of critical temperature. It means that one of two states (fragmental ring or continues ring) can been realized with high probabilities depending on the meaning of ratio $S_{\text {isl }} / S_{r}$ is larger or less than unity. It is seen that the state with the phase distribution in the form of fragments is more probable than the distribution in the form of the continuous ring at chosen values of parameters. In the vicinity to the critical temperature the fragmented ring is transformed into continues ring. Such transformation was observed in experiment [15]. 


\section{Conclusion}

In statistical theory of exciton condensed phase formation in two-dimensional system it is suggested that condensed phase arises as a result of attractive interaction between excitons. The investigated system is nonequilibrium one as a result of presence pumping and finite value of exciton lifetime. It forms some specific properties such as restricted sizes of condensed phase regions (islands), a correlation in positions of islands, so on. The theory was applied to explanation of experimental manifestation of condensed phase in quantum wells and also to explanation of the periodical fragmentation, which was observed in luminescence spectrum from a ring around a laser spot in a crystal with double quantum wells. For such explanations the theory does not require the exciton Bose-Einstein condensation.

1. S.V. Moskalenko, Bose-Einstain Condensation of Excitons and Biexciton, Academy of Science of Moldovian SSR, Kisheney (1970).

2. D. Hulin, A. Mysyrowicz, and a la Guillaume Benoitm, Phys. Rev. Lett. 45, 1970 (1980).

3. L. Keldysh, Excitons in Semiconductors, Nauka, Moscow (1971).

4. Ya. Pokrowskii, Phys. Status Solidi 11, 385 (1972).

5. T.M. Bragina, Yu.S. Lelikov, and Yu.G. Shreter, $Z h$. Exp. Theor. Phys. 79, 1838 (1980).

6. T.M. Rice, J.C. Hensel, T.G. Phillips, and G.A. Thomas, The Electron-Hole Liquid in Semiconductors, Academic Press, New York (1977).

7. N.V. Zamkovets, N.N. Sybeldin, and V.A. Tsvetkov, Zh. Exp. Theor. Phys. 105, 1066 (1994).

8. K.E. O'Hara and J.P. Wolfe, Phys. Rev. B62, 12909 (2000).

9. A.V. Larionov and V.B. Timofeev, JETP Lett. 73, 342 (2001); V.B. Timofeev, Usp. Fiz. Nauk 125, 315 (2005).
10. V. Krivolapchuk, E.S. Moskalenko, and A.L. Zhmodikov, Phys. Rev. B64, 045313 (2001).

11. L.V. Butov, A.C. Gossard, and D.S. Chemla, Nature 418, 751 (2002).

12. L.V. Butov, Solid State Commun. 127, 89 (2003).

13. D. Snoke, S. Denev, Y. Liu, L. Pfeiffer, and K. West, Nature 418, 754 (2002).

14. D. Snoke, Y. Liu, S. Denev, L. Pfeiffer, and K. West, Solid State Commun. 127, 187 (2003).

15. L.V. Butov, V.B. Levitov, A.V. Mintsev, B.D. Simons, A.C. Gossard, and D.S. Chemla, Phys. Rev. Lett. 92, 117404 (2004).

16. R. Rapaport, G. Chen, D. Snoke, S.H. Simon, L. Pfeiffer, K. West, Y. Liu, and S. Denev, Phys. Rev. Lett. 92, 117405 (2004).

17. O.L. Berman, Yu.E. Lozovik, D.W. Snoke, and R.D. Coalson, Phys. Rev. B70, 235310 (2004).

18. E.A. Cornell and C.E. Wieman, Rev. Mod. Phys. 74, 875 (2002).

19. D. Birkedal, J. Singh, V.G. Lyssenko, J. Erland, and J.M. Hvam, Phys. Rev. Lett. 76, 672 (1996).

20. Yu.E. Lozovik and O.L. Berman, JETP Lett. 64, 573 (1996).

21. Yu.E. Lozovik and O.L. Berman, JETP 84, 1027 (1997).

22. J. Fernandez-Rossier and C. Tejedor, Phys. Rev. Lett. 78, 4809 (1997).

23. I.M. Lifshits and V.V. Slyosov, J. Phys. Chem. Solids 19, 35 (1961).

24. R.N. Silver, Phys. Rev. B11, 1569 (1975).

25. P.M. Westerwelt, Phys. Status Solidi 74, 727 (1976).

26. V.S. Bagaev, N.V. Zamkovets, L.V. Keldysh, N.M. Sibeldin, and V.A. Tsetkov, JETP 70, 1501 (1976).

27. V.I. Sugakov, Mol. Cryst. Liq. Cryst. 384, 11 (2002).

28. V.I. Sugakov, Phys. Solid State 46, 1496 (2004).

29. V.I. Sugakov, Ukr. J. Phys. 49, 1117 (2004).

30. V.I. Sugakov, Solid State Commun. 134, 63 (2005). 